\title{
Nonhomologous end-joining deficiency of L5178Y-S cells is not associated with mutation in the ABCDE autophosphorylation cluster
}

\author{
Kamil Brzóska, Marcin Kruszewski and Irena Szumiel ${ }^{\circledR}$ \\ Department of Radiobiology and Health Protection, Institute of Nuclear Chemistry and Technology, \\ Warszawa, Poland; ${ }^{\circledR}$-mail: izasz@ichtj.waw.pl
}

Received: 31 August, 2005; revised: 12 October, 2005; accepted: 24 October, 2005 available on-line: 09 January, 2006

\begin{abstract}
Cells with mutated autophosphorylation sites in the ABCDE cluster of DNA-dependent protein kinase catalytic subunit (DNA-PKcs) are defective in the repair of ionising radiation-induced DSB, but show in an in vitro test the same DNA-PK activity as the cells possessing wild type enzyme. Nevertheless, the mutated DNA-PK is able to undergo ATP-dependent autophosphorylation and inactivation. This characteristics correspond well with the phenotypic features of the L5178Y-S (LY-S) cell line that is defective in DSB repair, shows a pronounced G1 phase radiosensitivity, but in which the level of DNA-PK activity present in total cell extracts is similar to that of its radioresistant counterpart L5178Y-R (LY-R) cell line. The purpose of this work was to examine the possible alterations in the sequence encoding the cluster of autophosphorylation sites in the DNA-dependent protein kinase in LY-S cells. Despite the presence of phenotypic features indicating the possibility of such alterations, no differences were found between the sequences coding for the autophosphorylation sites in L5178Y-R and L5178Y-S cells. In conclusion, the repair defect in LY-S cells is not related to the structure of the DNA-PK autophosphorylation sites
\end{abstract} (ABCDE casette).

Keywords: double strand break repair, mouse lymphoma L5178Y, DNA-dependent protein kinase, autophosphorylation, nonhomologous end-joining

The ionising radiation-hypersensitive L5178Y$S$ (LY-S) cell line was isolated from the parental L5178Y line (later called LY-R) and characterised by Alexander and Mikulski (1961). The 40-year studies on these cells have recently been reviewed (Szumiel, 2005a; 2005b). Although it is clear that the reason for the radiation sensitivity of LY-S cells is an impaired DNA DSB repair (Evans et al., 1987; Wlodek \& Hittelman, 1987; 1988b), the molecular defect remains unknown.

Two phenotypic features are characteristic for cells with defective NHEJ: a slowed down DSB repair and a pronounced G1 phase radiosensitivity. LY-S cells show both features. Impaired DSB repair was shown, among others, by neutral elution and premature chromosome condensation (Wlodek \& Hittelman, 1988b). Also, post-irradiation DNA repair in these cells is refractory to the DNA-PK specific inhibitor OK-1035, in contrast with that in the DSB repair-competent LY-R cells (Kruszewski et al., 1998). Nevertheless, in spite of the functional impairment in LY-S cells, the same level of DNA-PK activity is present in total cell extracts of both LY cell lines (Wojewodzka et al., 2004). This result indicates that the NHEJ defect in LY-S cells is not due to DNA-PK mutation that would affect its activity in vitro, as is the case in other radiation sensitive mammalian cell mutants. On the other hand, the cell-age dependence of survival (determination in subpopulations separated by elutriation (Wlodek \& Hittelman, 1988a) indicates a very marked radiosensitivity of G1 phase cells, typical for cells with NHEJ defect (Rothkamm et al., 2003).

Recent studies by Block et al. (2004) have indicated that cells expressing DNA-PKcs with mutated autophosphorylation sites in the so-called ABCDE cluster of serine and threonine residues in the central part of the molecule are defective in the repair

Abreviations: C1D, DNA binding protein C1D; DIR1, FK506 binding protein like, FKBPL; DNA-PKcs, DNA-dependent protein kinase catalytic subunit (EC 2.7.1.37); DSB, double strand break; EGFR, epidermal growth factor receptor; KUBs, Ku70-binding proteins; LY, L5178Y; NHEJ, nonhomologous end-joining; Sir, silent information regulator; Xrcc4, X-ray repair complementing defective repair in Chinese hamster cells 4 . 
of ionising radiation-induced DSB. Purified mutated DNA-PK proteins, however, showed in an in vitro test the same protein kinase activity as the wild type enzyme and were able to undergo ATP-dependent autophosphorylation and inactivation. Thus, the properties of mutants corresponded with those of LY-S cells.

It seemed plausible that such an autophosphorylation defect, which does not affect the kinase activity but is a cause of failure in DNA end-joining, is the cause of impaired NHEJ in LY-S cells. Therefore, we examined the Prkdc gene fragment encoding the cluster of autophosphorylation sites (ABCDE casette) in search for possible mutations that would affect autophosphorylation of DNA-PK in L5178Y-S (LY-S) cells. LY-R cells served as reference. No differences were found between the autophosphorylation sites in LY-R and LY-S cells, indicating that the defect in NHEJ in the latter cells is not related to the structure of the ABCDE casette.

\section{MATERIALS AND METHODS}

Cell cultures. Murine leukemic lymphoblast LY-R and LY-S were maintained in suspension cultures in RPMI 1640 medium (Sigma) supplemented with $8 \%$ heat-inactivated bovine serum and antibiotics (Szumiel, 1979).

RNA extraction, reverse transcriptionpolymerase chain reaction (RT-PCR) and sequencing. Total RNA was extracted using RNeasy Protect Mini Kit (Qiagen) according to the manufacturer's protocol. Two primers were designed. Primer 7627F with the sequence TCCCCCAAGATAGAAGTCCAC and primer 7977R - GATCCGCCAGTAGGTCAATG. First strand cDNA was synthesized from total RNA using Thermoscript RT-PCR System (Invitrogen) and primer $7977 \mathrm{R}$ at $50^{\circ} \mathrm{C}$ for $1 \mathrm{~h}$. Target sequence was amplified using primers $7627 \mathrm{~F}$ and 7977R by Platinum Taq DNA Polymerase (Invitrogen). The PCR protocol consisted of $3 \mathrm{~min}$ of denaturation at $94^{\circ} \mathrm{C}$, followed by five initial cycles comprising $30 \mathrm{~s}$ at $94^{\circ} \mathrm{C}, 2 \mathrm{~min}$ at $54.4^{\circ} \mathrm{C}$ and $45 \mathrm{~s}$ at $72^{\circ} \mathrm{C}$, then by 30 cycles comprising $30 \mathrm{~s}$ at $94^{\circ} \mathrm{C}$, $30 \mathrm{~s}$ at $54.4^{\circ} \mathrm{C}$ and $45 \mathrm{~s}$ at $72^{\circ} \mathrm{C}$. The final extension step was performed for $7 \mathrm{~min}$ at $72^{\circ} \mathrm{C}$. Amplification reactions were carried out in a PTC-200 DNA Engine Peltier Thermal Cycler (MJ Research). PCR products were sized by agarose electrophoresis and gel purifed using QIAquick Gel Extraction Kit (Qiagen). PCR products were sequenced using primers described above, BigDye v1.1 Terminator Cycle Sequencing Kit (Applied Biosystems) and ABI PRISM 377 DNA sequenator (Applied Biosystems).

Sequence analysis. LY-R and LY-S sequences were aligned and analysed using Bioedit 7.0.4.1 program (Hall, 1999) together with murine DNAPK nucleotide sequences obtained from GenBank (accession numbers: D87521, AB007544, AB011543, AB030754).

\section{RESULTS AND DISCUSSION}

In mammalian cells the DNA-PK dependent NHEJ is an important DSB repair system. Nevertheless, its basic level of activity very often does not correlate with radiosensitivity, as observed in screening experiments carried out in various types of cancer cells (Allalunis-Turner et al., 1995) in contrast with a very good correlation between radiosensitivity and residual DSB level. This result can be taken as an indication that (apart from obvious dysfunctions due to mutations) NHEJ activity (essential for repair in G0 and G1 phase cells) is subject to control at various levels and its efficiency may depend on various controlling mechanisms. To list only a few - stimulation of transcription of genes coding for the components of the system, proper timing of translocation to the nucleus, post-translational modifications, with phosphorylation being the most prominent and best known, binding of specific proteins (Artemis, KUBs, C1D). A single mechanism or several combined may modulate the efficiency of NHEJ and thus modify the cellular response to DNA damage inflicted by ionising radiation or chemicals.

In this work, we examined the DNA-PKcs (PRKDC) gene fragment encoding the cluster of autophosphorylation sites (ABCDE casette) in search for possible mutations that would affect the activity of DNA-PK in L5178Y cells. In spite of phenotypic features suggesting the presence of such mutations, no differences were found in the sizes of RT-PCR

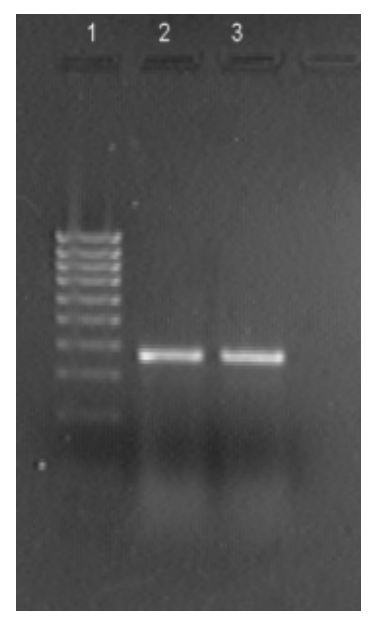

Figure 1. RT-PCR products of DNA-PKcs fragment (nt 7648-7976) containing ABCDE autophosphorylation cluster of LY-S and LY-R cells.

Lane 1, Gene Ruller® 100 bp DNA ladder (Fermentas); lane 2, LY-R cells; lane 3, LY-S cells. 

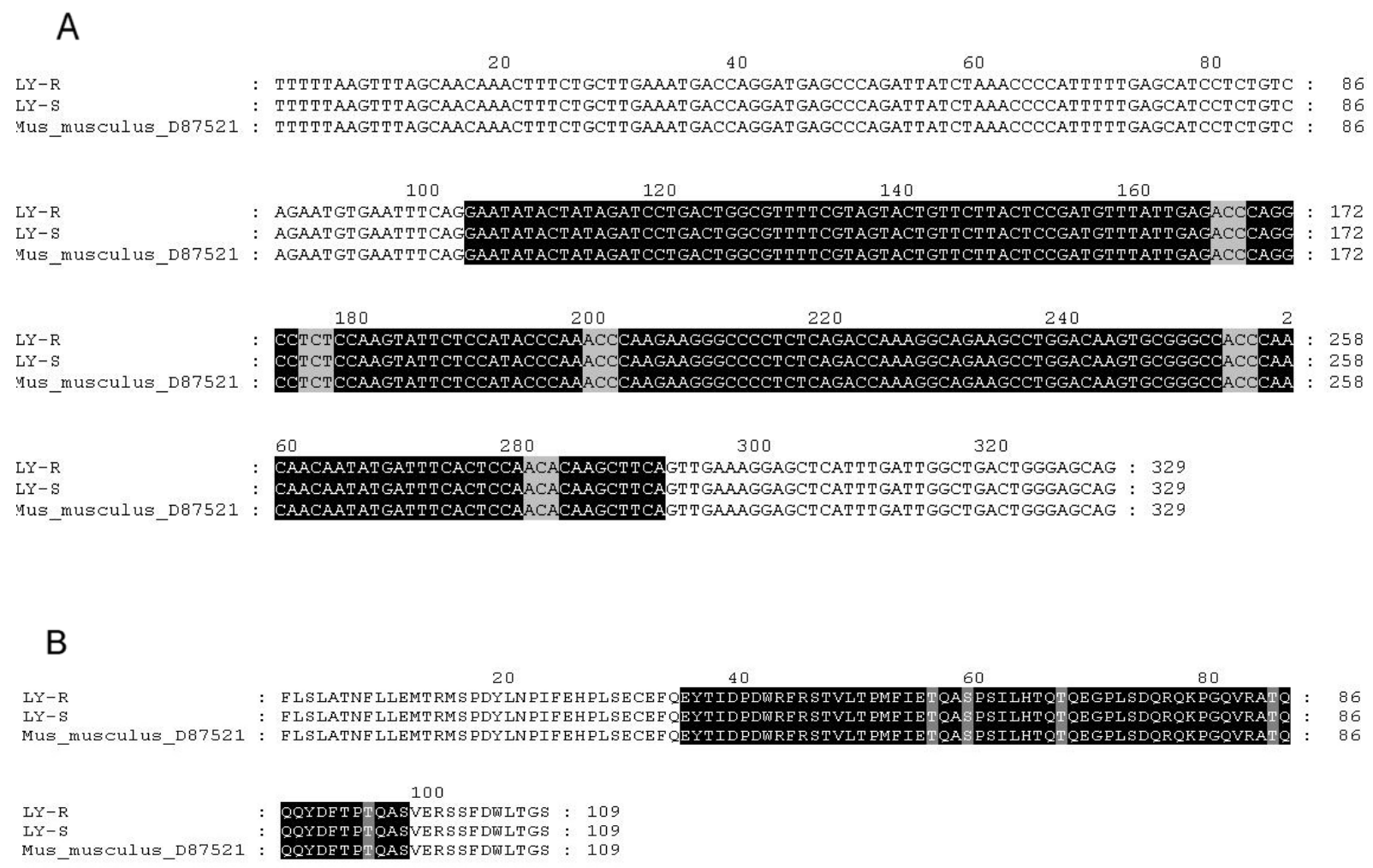

Figure 2. Nucleotide (A) and predicted amino-acid (B) sequences of DNA-PKcs region containing ABCDE autophosphorylation cluster of LY-S and LY-R cells compared with sequence of murine DNA-PKcs ABCDE autophosphorylation cluster (GenBank accesion No. D87521).

Exon 58 of DNA-PKCs is highlighted by black background, the five phosphorylation sites present in murine ABCDE cluster are shown in gray. Nucleotide sequences of DNA-PKcs region containing ABCDE autophosphorylation cluster of LY-S and LY-R cells were deposited in the GenBank under the accession Nos DQ235257 and DQ235258, respectively.

products from the two types of cells (Fig. 1). This, however, does not exclude a point mutation that might cause a loss of autophosphorylation sites, thus we sequenced the region of interest. Again, no differences were found in the sequences of the RTPCR products obtained from LY-R and LY-S cells (Fig. 2).

Although an alteration in the autophoshorylation of the ABCDE casette would clearly explain the difference in radiosensitivity of LY-S and LY-R cells, its lack leaves open other possible reasons for the NHEJ impairment in LY-S cells. It is known that components of DNA-PK are localised in the cytoplasm and may fail to translocate to the nucleus; an example of such a cause of radiation sensitivity has been described for cells of Long-Evans Cinnamon rats (Okui et al., 2002); a similar effect can be obtained by treatment of X-irradiated cells with $0.5 \mathrm{M}$ $\mathrm{NaCl}$ (Endoh et al., 2001) or by blocking the EGFR with an antibody specific for the extracellular part of the EGFR molecule (Huang et al., 1999; Dittmann et al., 2005). This may point to the importance of the nuclear-cytoplasmic traffic in the development of the effective response to irradiation.
Another possibility is that DNA-PK activity in situ is repressed by an unknown factor. An example of such factor is the protein encoded by a novel stress gene, DIR1, with some similarities to immunophilins (Robson et al., 1999; 2000). So far, only its influence on single-strand break rejoining was stated (Robson et al., 2000). If this were the case, LY-S cells would lack the mechanism that - upon irradiation - causes dissociation of the inhibitory molecule, thus freeing the enzyme's catalytic subunit and enabling it to form the active complex with the $\mathrm{Ku}$ heterodimer. The possibility that other components of NHEJ are defective in LY-S cells has partly been eliminated by complementation studies carried out in Japan (Sato et al., 1995). These authors have shown that the defect is not in Xrcc4 or ligase IV. Other components of NHEJ that could be defective in LY-S cells, such as the neuroblast differentiation associated protein, Ahnak (also known as desmoyokin (Stiff et al., 2004), Artemis or Sir proteins are less probable, since the phenotypic features of such deficiencies are different from those seen in LY-S cells (see recent reviews in Lees-Miller \& Meek, 2003; Pfeiffer et al., 2004; Kruszewski \& Szumiel, 2005). 
It should be added that many types of cancer cells have been screened for DNA-PK activity in an attempt to find a correlation with their radiation sensitivity (e.g., Allalunis-Turner et al., 1995). No such correlation was found; possibly this was due to the fact that whole-cell extracts were used for in vitro DNA-PK activity tests. As discussed above, the nucleo-cytoplasmic translocations of the components of the repair systems may be an important factor in the cellular response to DNA damage. They may be a suitable target for radiosensitivity modifications less toxic for the organism: it would be sufficient to inhibit or delay such translocations for a relatively short time: $0.5 \mathrm{M} \mathrm{NaCl}$ treatment exerts its sensitising effect within $20 \mathrm{~min}$ (Endoh et al., 2001). So, unveiling the secret of the DSB repair defect in LY-S cells may prove of more general interest, rather than being of value only for hunters of murine DNA repair deficiencies.

\section{Acknowledgements}

This work was supported by the State Committee for Scientific Research Statutory Grant for the Institute of Nuclear Chemistry and Technology.

\section{REFERENCES}

Alexander P, Mikulski B (1961) Nature 192: 572-573.

Allalunis-Turner MJ, Lintott LG, Barron GM, Day RS III, Lees-Miller SP (1995) Cancer Res 55: 5200-5202.

Block WD, Yu Y, Merkle D, Gifford JL, Ding Q, Meek K, Lees-Miller SP (2004) Nucleic Acids Res 32: 4351-4357.
Dittmann K, Mayer C, Fehrenbacher B, Schaller M, Raju U, Milas L, Chen DJ, Kehlbach R, Rodemann HP (2005) J Biol Chem 280: 31182-31189.

Endoh D, Okui T, Kon Y, Hayashi M (2001) Radiat Res 155: 320-327.

Evans HH, Ricanati M, Horng MF (1987) Proc Natl Acad Sci USA 84: 7562-7566.

Hall TA (1999) Nucleic Acids Symp Ser 41: 95-98.

Huang SM, Bock JM, Harari PM (1999) Cancer Res 59: 1935-1940.

Kruszewski M, Szumiel I (2005) DNA Repair (Amst) 4: 1306-1313.

Kruszewski M, Wojewodzka M, Iwanenko T, Szumiel I, Okuyama A (1998) Mutat Res 409: 31-36.

Lees-Miller SP, Meek K (2003) Biochimie 85: 1161-1173.

Okui T, Endoh D, Kon Y, Hayashi M (2002) Radiat Res 157: 553-561.

Pfeiffer P, Goedecke W, Kuhfittig-Kulle S, Obe G (2004) Cytogenet Genome Res 104: 7-13.

Robson T, Joiner MC, Wilson GD, McCullough W, Price ME, Logan I, Jones H, McKeown SR, Hirst DG (1999) Radiat Res 152: 451-461.

Robson T, Price ME, Moore ML, Joiner MC, McKelveyMartin VJ, McKeown SR, Hirst DG (2000) Int J Radiat Biol 76: 617-623.

Rothkamm K, Kruger I, Thompson LH, Lobrich M (2003) Mol Cell Biol 23: 5706-5715.

Sato K, Chen DJ, Eguchi-Kasai K, Itsukaichi H, Odaka T, Strniste GF (1995) J Radiat Res 36: 38-45.

Stiff T, Shtivelman E, Jeggo P, Kysela B (2004) DNA Repair (Amst) 3: 245-256.

Szumiel I (1979) Chem-Biol Interact 24: 51-72.

Szumiel I (2005a) Int J Radiat Biol 81: 339-352.

Szumiel I (2005b) Int J Radiat Biol 81: 353-365.

Wlodek D, Hittelman WN (1987) Radiat Res 112: 146-155.

Wlodek D, Hittelman WN (1988a) Radiat Res 115: 550-565.

Wlodek D, Hittelman WN (1988b) Radiat Res 115: 566-575.

Wojewodzka M, Kruszewski M, Sochanowicz B, Szumiel I (2004) Int J Radiat Biol 80: 473-482. 